\title{
Percutaneous and surgical treatment of pyogenic liver abscesses: Observation over a 21 -year period in 148 patients
}

\author{
G. Ferraioli ${ }^{\text {a,* }}$, A. Garlaschelli ${ }^{\text {a }}$, D. Zanaboni ${ }^{\text {b }}$, R. Gulizia ${ }^{\text {a }}$, \\ E. Brunetti ${ }^{\text {a }}$, F.P. Tinozzi ${ }^{c}, 1$, C. Cammà ${ }^{d}$, C. Filice ${ }^{a}$ \\ a Infectious and Tropical Diseases Division, IRCCS S. Matteo, University of Pavia, \\ Via Taramelli 5, 27100 Pavia, Italy \\ b Infectious Diseases Division, IRCCS S. Matteo, University of Pavia, \\ Via Taramelli 5, 27100 Pavia, Italy \\ c Surgery Department, IRCCS S. Matteo, University of Pavia, Via Taramelli 5, 27100 Pavia, Italy \\ ${ }^{\mathrm{d}}$ Gastroenterology Unit, University of Palermo, Italy \\ Received 16 October 2007; accepted 29 January 2008
}

\begin{abstract}
Background. Percutaneous drainage of pyogenic liver abscess has become first-line treatment. In the past surgical drainage was preferred in some centres.

Aim. The aim of this retrospective study was to assess the effectiveness of percutaneous treatments and surgical drainage, in terms of treatment success, hospital stay and costs.

Patients. Data of 148 patients (90 males; 58 females; mean age, 61 yrs; range, 30-86 yrs) were retrospectively analysed.

Methods. Patients' outcomes, including the length of hospital stay, procedure-related complications, treatment failure and death, were recorded. Multiple logistic regression model was used for statistical analysis.

Results. One hundred and four patients ( 83 with solitary and 21 with multiple abscesses) were treated percutaneously, either by needle aspiration (91 patients) or catheter drainage (13 patients) depending on the abscess's size, and 44 patients ( 30 with solitary and 14 with multiple abscesses) were treated surgically. There was no statistically significant difference in patients' demographics or abscess characteristics between groups. Hospital stay was longer, and costs were higher in patients treated surgically $(p<0.001)$. There was statistically significant difference in morbidity rate between groups $(p<0.001)$. No death occurred in both groups.

Conclusions. Percutaneous and surgical treatment of pyogenic liver abscesses are both effective, nevertheless percutaneous drainage carries lower morbidity and is cheaper.

(C) 2008 Published by Elsevier Ltd on behalf of Editrice Gastroenterologica Italiana S.r.l.
\end{abstract}

Keywords: Drainage; Interventional procedure; Liver; Liver abscess; Method $\overline{\bar{\nu}}$ genic; Retrospective studies; Treatment outcome; Ultrasound

\section{Introduction}

Pyogenic liver abscess (PLA) is a serious, life threatening condition, with a mortality rate of $6-14 \%[1,2]$. In the antibiotics era the most $\equiv$ non pre $\equiv$ sing factor has become biliary tract disease

\footnotetext{
* Corresponding author. Tel.: +390382 502799; fax: +390382 301987.

E-mail address: ferraiol@tin.it (G. Ferraioli).

${ }^{1}$ Present address: General Surgery Division, Lodi Hospital, Lodi (MI), Italy.
}

aetiological agents commonly represented by Gram-negative and anaerobic bacteria $[3,4]$.

Other facilitating conditions are systemic infections through haematogenous dissemination, penetrating traumas, and abdominal surgical procedures in particular in patients with impaired immunity due to malignancies, AIDS, diabetes, etc. $[2,3,5,6]$.

Therapeutic options are medical therapy alone, reserved to few cases of multiple small pus collections [6,7], and percutaneous or surgical drainage both in combination with appropriate intravenous antibiotics. Over the last two

1590-8658/\$30 @ 2008 Published by Elsevier Ltd on behalf of Editrice Gastroenterologica Italiana S.r.1. doi:10.1016/j.dld.2008.01.016 
decades, percutaneous drainage has become the first-line treatment in most cases [1,8-12], even though surgical drainage is more likely to be required in patients with abscess rupture, incomplete percutaneous drainage or who have uncorrected primary pathology [7].

In the past surgical drainage was preferred in some centres [13]. A recent study has shown that for liver abscesses larger than $5 \mathrm{~cm}$ surgical drainage provides better clinical outcomes than percutaneous drainage with comparable morbidity and mortality rates [14].

The aims of this study were to retrospectively analyse the clinical course of patients with PLA treated with percutaneous or surgical drainage in a different period of time and to assess the effectiveness of both treatments in terms of success, number of secondary procedures, hospital stay, morbidity and mortality. In addition, comparison between the two groups was made and the benefits of both procedures in relation to their costs were retrospectively analysed.

\section{Patients and methods}

\subsection{Patients}

Data of patients admitted to two Departments of Infectious Diseases at two different institutions, San Matteo Hospital in Pavia and Cotugno Hospital in Naples, from January 1998 to December 2002 were retrospectively collected. Besides, data of patients admitted to the Department of Surgery at San Matteo Hospital in Pavia from November 1981 to December 2002 were also collected. The medical files of 163 patients affected by PLA treated either percutaneously or surgically were retrieved. Amoebic abscesses and hydatid liver cysts were actively excluded. Fifteen patients with PLA were excluded from the analysis because some data were missing.

Thus, our study cohort consisted of 148 patients ( 90 males; 58 females; mean age, 61 yrs; range, 30-86 yrs). In particular, 79 patients (47 males; 32 females; mean age, 62 yrs; range, 39-86 yrs) were treated at the Department of Infectious Diseases of Cotugno Hospital, 25 patients (16 males; 9 females; mean age, 63 yrs; range, 30-80 yrs) at the Department of Infectious Diseases of San Matteo Hospital, and 44 patients (27 males; 17 females; mean age, 57 yrs; range, 32-82 yrs) at the Department of Surgery of San Matteo Hospital.

The diagnosis of PLA was made by ultrasonography (US) alone in 118 patients, by US and computed tomography in 25 patients, by computed tomography alone in four patients, and by magnetic resonance imaging alone in one patient.

All 104 patients (63 males; 41 females; mean age, 63 yrs; range, 30-86 yrs) from the two Departments of Infectious Diseases were treated percutaneously (PT), either by fineneedle aspiration (NA) or catheter drainage (CD), depending on abscesses size. The 44 patients from the Department of Surgery of San Matteo Hospital were treated surgically (ST).

\subsection{Methods}

This retrospective study was approved by the institutional ethics review board of both hospitals that waived the requirement for informed consent.

Underlying pathologies, size and number of the abscesses were determined. In case of multiple abscesses, the size of the largest one was estimated.

Patients' outcomes, including the length of hospital stay, procedure-related complications, treatment failure and death, were recorded.

\subsubsection{Percutaneous treatments}

Both NA and CD were performed under US guidance. The US systems used during the study period were Aloka Prosound SSD-5000 (Tokyo, Japan) with a 3.0-6.0 MHz convex array broadband probe and a $3.5 \mathrm{MHz}$ microconvex probe at the Department of Infectious Diseases of Cotugno Hospital, and AU5 Harmonic (ESAOTE, Genoa, Italy) equipped with a $3.5-5.0 \mathrm{MHz}$ convex array probe at Department of Infectious Diseases of San Matteo Hospital.

The freehand technique was used both at Cotugno Hospital and at San Matteo Hospital.

On both institutions the methodologies for percutaneous needle aspiration and catheter drainage elsewhere described were followed $[6,15]$.

As a rule, fine-needle aspiration was used for abscesses up to $6 \mathrm{~cm}$ in size. Catheter from 8 to $10 \mathrm{~F}$ was positioned in case of abscesses larger than $6 \mathrm{~cm}$, and removed when no more than 10-20 $\mathrm{mL}$ of material was drained during a period of $24 \mathrm{~h}$.

\subsubsection{Surgical treatment}

Surgical drainage was performed as the first-line treatment due to the choice of the surgical team until the year 2000, afterwards there was a case discussion with the team of infectious diseases units. In case of failure of surgical drainage, hepatic resection was executed.

Either in patients treated percutaneously or surgically, aerobic, anaerobic and fungal cultures were performed on the aspirated material, accompanied by sensitivity testing. Systemic broad-spectrum antibiotics were administered until the results of pus culture were available. Afterwards, the most active antibiotic was given. In patients with a cytological diagnosis of abscess but negative findings of pus cultures, broad-spectrum antibiotic therapy was continued. The patients were discharged from the hospital on oral antibiotics, to complete a 6-week course from the time of procedure, when fever and clinical signs disappeared and the abscess' dimensions were reduced to at least one-third of the initial size on US examination.

\subsection{Variables}

We considered sex, age, number and size of abscess, relapses and length of hospital stay. Besides, based on 
duration of hospital stay and therapeutic procedures, we calculated costs.

\subsection{Costs}

The costs were calculated using diagnosis related group (DRG) system, a method currently used in the USA and in some European countries to reimburse hospitals. The DRG system classifies hospital cases into approximately 500 groups, each one expected to consume similar hospital resource. The effective reimbursement is fixed for groups of pathology, related to a chosen treatment, and increases in case of complications. In the DRG system the hospitalization length is established according to procedures used.

In the reimbursement scheme adopted in our country, there is no difference between hepatic resection and surgical drainage. Thus, costs are unaffected by different surgical procedures.

\subsection{Follow-up}

The follow-up was based on US and clinical examinations performed in all patients every 3 days during the first week, monthly for the first 6 months after the procedure, and every 6 months thereafter. Follow-up studies were terminated when findings of two consecutive US examinations showed complete healing of the liver parenchyma.

\subsection{Statistical analysis}

Continuous variables were summarized as frequency and percentage. Multiple logistic regression model was used to assess the relationship between length of hospitalization and demographic and clinical characteristics of the patients. In the model the dependent variable was length of hospital stay coded as 0 when shorter or equal to 14 days (short hospitalization) or as 1 when longer than 14 days (long hospitalization). As candidate risk factors for longer hospitalization we considered age, sex, abscess number, size, and percutaneous or surgical treatment.

Variables found to be associated to the dependent variables on univariate logistic regression at a probability threshold $<0.10$ were included in multivariate logistic regression models.

Regression analysis was performed using PROC LOGISTIC sub routine in SAS (SAS Institute, Inc. Cary, North Carolina, USA). A $p$ value less than 0.05 was considered to indicate a statistically significant difference.

\section{Results}

Two hundred and five PLA were found in 148 patients. The results of pus cultures are reported in Table 1 .

Thirty-five of 148 patients (23.6\%) had multiple liver abscesses (range 2-8).
Table 1

Results of pus cultures in 148 patients with 205 pyogenic liver abscesses

\begin{tabular}{lccc}
\hline Results of pus culture & PT & ST & Overall \\
\hline Aerobic organism & & & \\
$\quad$ Escherichia coli & $24(23.1)$ & $13(29.5)$ & $37(25.0)$ \\
Staphylococcus aureus & $8(7.7)$ & $4(9.1)$ & $12(8.1)$ \\
Klebsiella & $8(7.7)$ & $2(4.6)$ & $10(6.8)$ \\
Enterobacter & - & $1(2.3)$ & $1(0.7)$ \\
Anaerobic organism & & & \\
$\quad$ Peptostreptococcus spp. & $10(9.6)$ & $4(9.1)$ & $14(9.4)$ \\
Bacteroides & $8(7.7)$ & - & $8(5.4)$ \\
Fusobacterium & $2(1.9)$ & - & $2(1.4)$ \\
Others & $9(8.7)$ & $3(6.8)$ & $12(8.1)$ \\
Mixed & $12(11.5)$ & $4(9.1)$ & $16(10.8)$ \\
Negative & $23(22.1)$ & $13(29.5)$ & $36(24.3)$ \\
Total & 104 & 44 & 148
\end{tabular}

PT: percutaneously treated patients; ST: surgically treated patients. Figures in parenthesis are percentages.

Table 2

Underlying pathology of the 148 patients with 205 pyogenic liver abscesses

\begin{tabular}{lclc}
\hline Underlying pathology & PT & ST & Overall \\
& No. $(\%)$ & No. $(\%)$ & No. $(\%)$ \\
\hline Biliary tract diseases & $17(16.3)$ & $12(27.3)$ & $29(19.6)$ \\
Acute cholecystitis & $5(4.8)$ & $4(9.1)$ & $9(6.1)$ \\
\multicolumn{1}{c}{ Ascending cholangitis } & $12(11.5)$ & $8(18.2)$ & $20(13.5)$ \\
Malignancies & $36(34.6)$ & $16(36.4)$ & $52(35.1)$ \\
AIDS & $8(7.7)$ & - & $8(5.4)$ \\
Diabetes & $9(8.7)$ & $6(13.6)$ & $15(10.1)$ \\
Previous abdominal surgery & $9(8.7)$ & - & $9(6.1)$ \\
Others & $22(21.1)$ & $9(20.4)$ & $31(21.0)$ \\
Cryptogenic & $3(2.9)$ & $1(2.3)$ & $4(2.7)$ \\
Total & 104 & 44 & 148 \\
\hline
\end{tabular}

PT: percutaneously treated patients; ST: surgically treated patients. Figures in parenthesis are percentages.

The mean size of the abscesses was $5.2 \mathrm{~cm}$ (range $1.7-12.0 \mathrm{~cm})$.

Underlying pathologies of abscess formation are reported in Table 2. A potential underlying pathology was found in 144 of 148 patients (97.3\%). In 21 of 52 patients (40.4\%) with malignancies, the primary site was the biliary tract. Eleven of 29 patients (37.9\%) with biliary diseases had had a prior biliary procedure.

Patients' clinical characteristics on admission are reported in Table 3. No septic condition was recorded in our cohorts.

Table 3

Clinical characteristics on admission in the 148 patients with 205 pyogenic liver abscesses

\begin{tabular}{lc}
\hline Symptoms and signs & No. of patients $(\%)$ \\
\hline Fever & $133(89.9)$ \\
Nausea & $101(68.2)$ \\
Abdominal pain & $59(39.9)$ \\
Jaundice & $30(20.3)$ \\
Hepatomegaly & $77(52.0)$ \\
\hline
\end{tabular}

Figures in parenthesis are percentages. 
Table 4

Variable analysed in the 148 patients with 205 pyogenic liver abscesses

\begin{tabular}{|c|c|c|c|c|}
\hline Variable & All & PT $(\%)$ & $\mathrm{ST}(\%)$ & Statistical significance \\
\hline Patients no. & 148 & 104 & 44 & \\
\hline Mean age (yr) & 61 & 63 & 57 & ns \\
\hline \multicolumn{5}{|l|}{ Sex } \\
\hline Male & $90(60.8)$ & $63(60.5)$ & $27(61.3)$ & ns \\
\hline Female & $58(39.2)$ & $41(39.5)$ & $17(38.7)$ & ns \\
\hline Patients with solitary abscess & $113(76.4)$ & $83(79.8)$ & $30(68.2)$ & \\
\hline Patients with multiple abscesses & $35(23.6)$ & $21(20.2)$ & $14(31.8)$ & \\
\hline Abscess mean size $(\mathrm{cm})$ & 5.2 & 5.1 & 5.3 & ns \\
\hline \multicolumn{5}{|l|}{ Hospital stay in days (range) } \\
\hline All patients & $10.5(3-38)$ & $5.7(3-18)$ & $19.0(6-38)$ & 0.001 \\
\hline Patients with solitary abscess & $9.8(3-38)$ & $5.6(3-10)$ & $19.2(6-38)$ & 0.001 \\
\hline Patients with multiple abscesses & $13.2(3-31)$ & $6.7(3-18)$ & $18.7(11-31)$ & 0.001 \\
\hline
\end{tabular}

PT: percutaneously treated patients; ST: surgically treated patients. Figures in parenthesis are percentages. Statistical significance: PT versus ST.

\subsection{Percutaneous treatments group}

A hundred and four patients with 138 PLA were treated percutaneously. Eighty-three of 104 patients (79.8\%) had solitary and 21 of 104 patients (20.2\%) multiple PLAs. The mean diameter of PLA was $5.1 \mathrm{~cm}$ (range, $1.7-12.0 \mathrm{~cm}$ ) (Table 4).

No statistically significant difference was found between patients from the two different departments of infectious diseases in terms of clinical data and abscess characteristics.

The length of hospital stay ranged from 3 to 10 days (mean, 5.6 days) for patients with solitary PLA, and from 3 to 18 days (mean, 6.7 days) for patients with multiple PLA, the difference did not reach statistical significance. A hundred and three of 104 patients (99.0\%) had a hospital stay duration of less than 14 days.

No death occurred. No procedure-related complications occurred.

Ninety-one of 104 patients $(87.5 \%)$ were treated by NA, and 13 of 104 patients $(12.5 \%)$ by CD. Abscesses treated with $\mathrm{CD}$ were larger than those treated with NA (mean size, $8.0 \mathrm{~cm}$; range $5.5-12.0 \mathrm{~cm}$; versus mean size, $4.3 \mathrm{~cm}$; range, $1.7-8.0 \mathrm{~cm})$

Eighty-five of $91(93.4 \%)$ patients were treated in a single session. In 66 of $85(77.6 \%)$ a single needle insertion was performed, whereas in 19 of $85(22.4 \%)$ more than one needle insertion was required. In 6/91 (6.6\%) patients, two sessions of NA were necessary because the fever did not remit, and the size of PLAs remained unchanged on US examination.

All percutaneous treatments were successful. None required surgery.

\subsection{Surgical treatment group}

Forty-four patients with 67 PLA were treated surgically. Thirty of 44 patients $(68.2 \%)$ had solitary and 14 of 44 patients (31.8\%) had multiple PLAs. The mean diameter was $5.3 \mathrm{~cm}$ (range, $2.0-12.0 \mathrm{~cm}$ ) (Table 4). Length of hospital stay ranged from 6 to 38 days (mean, 19.2 days) for patients with solitary PLA, and from 11 to 31 days (mean, 18.7 days) for patients with multiple PLA, without a statistically significant difference. Eleven of 44 patients $(25.0 \%)$ had a hospital stay of less than 14 days.

No death occurred. The overall morbidity rate was $9.1 \%$. Two patients experienced pneumonia, one pleural effusion requiring drainage, and one wound infection.

Surgical drainage was successful in 41 of 44 patients (93.2\%), but three of 41 patients $(7.3 \%)$ required a second catheter insertion because of incomplete drainage. Three of 44 patients $(6.8 \%)$ had hepatic resection because of surgical drainage failure. Two of them had single PLA and one multiple PLA. In these three patients, the abscesses size did not decrease and fever persisted despite antibiotics. They all had multiloculated abscesses containing thick material.

\subsection{Costs}

The PT costs per patient were Euro 1746.88 in case of uncomplicated PLA and Euro 3105.44 for the complicated one. The calculated reimbursement is the same for a hospital stay of 3-29 days. In our series of patients, no complications were recorded. Thus, the mean cost per patient was Euro 1746.88 .

Table 5

DRG reimbursement scheme for liver abscesses

\begin{tabular}{llc}
\hline Procedure & \multicolumn{2}{l}{ DRG reimbursement (in Euro) } \\
\cline { 2 - 3 } & $\begin{array}{l}\text { Uncomplicated } \\
\text { abscess }\end{array}$ & $\begin{array}{l}\text { Complicated } \\
\text { abscess }\end{array}$ \\
\hline Percutaneous treatment & $1,746.88$ & $3,105.44$ \\
Surgical drainage & $5,287.99$ & $17,101.00$ \\
Surgical resection & $5,287.99$ & $17,101.00$ \\
\hline
\end{tabular}


The costs of hepatic resection or surgical drainage with incision were Euro 5287.99 for uncomplicated cases, and Euro 17,101.00 for complicated cases. The mean cost per patient was Euro 6898.85 (Table 5).

\subsection{Comparison between percutaneous treatment $(n=104)$ and surgical treatment $(n=44)$ groups}

There was no statistically significant difference in patients' demographics of the two groups. AIDS was an underlying disease only in the PT group, whereas biliary tract diseases were more common in the ST group (27.3\% versus $16.3 \%$ ). Only patients in the PT group had previous abdominal surgery. No statistical difference in abscess size was observed. Multiple PLAs were found in $31.8 \%$ of cases in ST group and $20.2 \%$ in the PT group $(p<0.05)$ (Table 4). Within groups, there was no statistically significant difference in length of hospital stay between patients with solitary and with multiple PLA. There were three treatment failures in the ST group, and none in the PT group.

There was a statistically significant difference in the morbidity rate between the two groups $(p<0.001)$. Costs of PT were significantly lower than costs of ST $(p<0.001)$.

From the multivariate analysis, surgical therapy was the only predictor of long hospitalization ( $>14$ days).

\subsection{Follow-up}

Complete resolution of parenchymal lesions at imaging was observed within maximum 86 days (range, 44-86 days; mean, 56 days) after discharge from the hospital. During the follow-up, (range, 4-12 months; mean, 9 months) no recurrence of abscesses was observed in both groups. No differences were observed between the two groups in terms of time to healing.

Eight patients were lost to follow-up before complete healing of liver abscess was achieved.

\section{Discussion}

In the last decades, the treatment and prognosis of pyogenic liver abscesses have significantly improved [16]. The use of new antimicrobials, advancements in diagnostic imaging techniques, and percutaneous image-guided drainage procedures are the main factors accounting for the observed improvements $[1,9,11,16,17]$. Nowadays, imageguided percutaneous drainage is the treatment of choice of PLA. It must be available in every hepato-biliary unit where a close cooperation between surgeons and interventional radiologists usually exists. The presence of a coexisting primary intra-abdominal pathology, the success rate of the procedure and expertise in managing PLA are factors to be considered in selecting the choice of therapy [1]. Surgery is reserved to severe cases, such as operative treatment of causative underlying diseases. Thus, percutaneous and surgical techniques are not competing methods, but have different indications and surgery also represents an option for non-responders to percutaneous treatment.

In this retrospective study, the results obtained in the two groups are not completely comparable because they were observed in different period of time. Nonetheless, they show that, independently from the management strategy used for PLA treatment, all patients recovered. The patients in the ST group had a significantly longer duration of hospital stay. Even though the rate of multiple PLAs was significantly higher in the ST group compared to the PT group the abscesses number did not significantly affect the length of hospital stay. Patients with multiple PLA had a shorter hospitalization in the PT group compared to ST group. The effectiveness of percutaneous drainage of multiple PLA was similar to that of surgery. This result is in keeping with other published studies [18,19], and suggest that percutaneous drainage should always be undertaken before surgery, the latter being reserved to selected cases such as those in which the percutaneous option has failed or with large multiloculated abscesses containing thick pus or when an underlying pathology requires operation. In our study, none patients in the PT group had underlying pathologies needing a not deferrable surgical treatment.

There are dissimilarities in patients' population between our series and that of other studies [2,3,5]. In our series, malignancy was the most frequent underlying pathology in both groups. Moreover, 8 of 104 patients (7.7\%) in the PT group had AIDS. Both conditions might be associated with a poor prognosis $[6,16]$. Nonetheless, no death occurred during the hospitalization and the follow-up period. Besides, in our series of patients there were nine cases of postsurgical PLA in the PT group. These differences in underlying pathologies did not lead to a longer duration of hospital stay compared to literature data $[1,18]$. In our series the mean follow up period was 9 months and it was tailored to liver abscess healing and not to underlying pathologies. It was terminated when findings of two consecutive US examinations showed complete healing of the liver parenchyma. Eight patients were lost to follow up before complete healing of liver abscess was achieved. Taking this into account makes our results less surprising and more in keeping with the available literature. Gram-negative aerobes were the major abscess pathogens in our series. Similar to other reports $[3,13,18]$, Escherichia coli was the most common pathogen, with a percentage of $25 \%$ of cases. The spectrum of causative organisms is similar to other European studies, but in Asian countries other distributions are seen (with high proportions of Klebsiella) [20]. The comparability with these patients' population is therefore limited. In $24.3 \%$ of cases, the results of pus culture were negative. This finding might be due to antimicrobial therapy regimen before hospitalization. 
No significant difference was found between the PT and ST group in terms of treatment success rate, suggesting that both procedure are effective.

Nonetheless, the aggressiveness of surgery should be taken into account. Patients in the ST showed a longer length of hospital stay and a higher morbidity rate.

This being a retrospective study, the selection criteria were also a function of time period considered; specifically, PTs were chosen where and when available (i.e. in the two departments of infectious diseases they were considered first choice based on the available local expertise) whereas surgical drainage was chosen by local surgeons as a first-line treatment, based on their own expertise and the time of intervention. This was 1981-2002, which includes a decade when PTs were not as popular as they became later on.

Over the last two decades, open surgical drainage has been increasingly been reserved to patients unsuitable for percutaneous drainage, the latter being safe, less invasive, more acceptable to patients and cheaper.

Nowadays, besides effectiveness of a treatment and reduction of patient discomfort, procedure-related costs is another issue that should be taken into account. Yet, to our knowledge, an analysis of costs of PT and ST has never been attempted. In a world of limited health care resources, the benefits of an intervention in relation to its costs should be considered, in order to balance costs against clinical outcomes. Economic evaluations are increasingly common in the medical literature [21-23]. Assigning a monetary value to a service or consumed resource is not trivial because clinicians should be aware not only of the clinical benefits of a particular strategy, but of the economic consequences as well.

All but one patient in the PT group had a hospitalization shorter than 14 days and the statistical analysis showed that the only factor influencing length of hospitalization was the type of treatment (ST or PT).

There are a number of limitations to our study. First, it was retrospective and the analysis of costs could only be based on DRGs. Second, costs presented in this study were not adjusted to the medical care costs of the study period. We did not think that this adjustment was necessary because changes in costs would have the same effect on both procedures.

Third, the number of patients in the surgically treated series is different from that of the percutaneously treated series. This is because percutaneous drainage has become the preferred choice of PLA treatment in the last decade.

The results of this study confirm that percutaneous and surgical treatment of pyogenic liver abscesses are both effective, but percutaneous drainage carries lower morbidity and is cheaper. In similar clinical settings, percutaneous drainage of liver abscesses should always be attempted as the first choice treatment, and it might help to optimise clinical conditions prior to surgery.

\section{Practice points}

- In our series Gram-negative aerobes were the major abscess pathogens. Escherichia coli was the most common pathogen, with a percentage of $25 \%$ of cases.

- The results of our study suggest that percutaneous drainage should always be undertaken before surgery, the latter being reserved to selected cases such as those in which the percutaneous option has failed or with large multiloculated abscesses containing thick pus or when an underlying surgical pathology requires operation.

- Percutaneous drainage must be available in every hepato-biliary unit where a close cooperation between surgeons and interventional radiologists usually exists.

\section{Research agenda}

- Percutaneous drainage of liver abscesses should always be attempted as the first choice treatment, and it might help to optimise clinical conditions prior to surgery.

- Prospective studies on comparative analysis of procedure-related costs are needed.

\section{Conflict of interest statement}

None declared.

\section{Acknowledgements}

The authors express their appreciation to all the investigators who collected the data presented in this article. In particular, they acknowledge the contribution of all the doctors of the Ultrasound Interventional Service of D. Cotugno Hospital, Naples, and all of the surgeons of the Surgical Divisions of S. Matteo Hospital, Pavia.

\section{References}

[1] Yu SC, Ho SS, Lau WY, Yeung DT, Yuen EH, Lee PS, et al. Treatment of pyogenic liver abscess: prospective randomized comparison of catheter drainage and needle aspiration. Hepatology 2004;39:932-8.

[2] Alvarez Perez JA, Gonzalez JJ, Baldonedo RF, Sanz L, Carreno G, Junco A, et al. Clinical course, treatment, and multivariate analysis 
of risk factors for pyogenic liver abscess. Am J Surg 2001;181:17786.

[3] Rahimian J, Wilson T, Oram V, Holzman RS. Pyogenic liver abscess: recent trends in etiology and mortality. Clin Infect Dis 2004;39: 1654-9.

[4] Cerwenka H, Bacher H, Werkgartner G, El-Shabrawi A, Kornprat P, Bernhardt GA, et al. Treatment of patients with pyogenic liver abscess. Chemotherapy 2005;51:366-9.

[5] Johannsen EC, Sifri CD, Madoff LC. Pyogenic liver abscesses. Infect Dis Clin North Am 2000;14:547-63.

[6] Civardi G, Filice C, Caremani M, Giorgio A. Hepatic abscesses in immunocompromised patients: ultrasonically guided percutaneous drainage. Gastrointest Radiol 1992;17:175-8.

[7] Barakate MS, Stephen MS, Waugh RC, Gallagher PJ, Solomon MJ, Storey DW, et al. Pyogenic liver abscess: a review of 10 years' experience in management. Aust N Z J Surg 1999;69:205-9.

[8] Kuligowska E, Connors SK, Shapiro JH. Liver abscess: sonography in diagnosis and treatment. AJR Am J Roentgenol 1982;138:253-7.

[9] vanSonnenberg E, Wittich GR, Goodacre BW, Casola G, D’Agostino HB. Percutaneous abscess drainage: update. World J Surg 2001;25: 362-9.

[10] Ch Yu S, Hg Lo R, Kan PS, Metreweli C. Pyogenic liver abscess: treatment with needle aspiration. Clin Radiol 1997;52:912-6.

[11] Rintoul R, O’Riordain MG, Laurenson IF, Crosbie JL, Allan PL, Garden OJ. Changing management of pyogenic liver abscess. Br J Surg 1996;83:1215-8.

[12] Rajak CL, Gupta S, Jain S, Chawla Y, Gulati M, Suri S. Percutaneous treatment of liver abscesses: needle aspiration versus catheter drainage. AJR Am J Roentgenol 1998;170:1035-9.

[13] Chou FF, Sheen-Chen SM, Chen YS, Chen MC. Single and multiple pyogenic liver abscesses: clinical course, etiology, and results of treatment. World J Surg 1997;21:384-8.
[14] Tan YM, Chung AY, Chow PK, Cheow PC, Wong WK, Ooi LL, et al. An appraisal of surgical and percutaneous drainage for pyogenic liver abscesses larger than $5 \mathrm{~cm}$. Ann Surg 2005;241:485-90.

[15] Mueller PR, vanSonnenberg E, Ferrucci Jr JT. Percutaneous drainage of 250 abdominal abscesses and fluid collections. Part II: Current procedural concepts. Radiology 1984;151:343-7.

[16] Huang CJ, Pitt HA, Lipsett PA, Osterman Jr FA, Lillemoe KD, Cameron JL, et al. Pyogenic hepatic abscess. Changing trends over 42 years. Ann Surg 1996;223:600-7.

[17] Lee KT, Wong SR, Sheen PC. Pyogenic liver abscess: an audit of 10 years' experience and analysis of risk factors. Dig Surg 2001;18:459-65.

[18] Giorgio A, de Stefano G, Di Sarno A, Liorre G, Ferraioli G. Percutaneous needle aspiration of multiple pyogenic abscesses of the liver: 13-year single-center experience. AJR Am J Roentgenol 2006;187:1585-90.

[19] Tazawa J, Sakai Y, Maekawa S, Ishida Y, Maeda M, Marumo F, et al. Solitary and multiple pyogenic liver abscesses: characteristics of the patients and efficacy of percutaneous drainage. Am J Gastroenterol 1997;92:271-4.

[20] Yang CC, Yen CH, Ho MW, Wang JH. Comparison of pyogenic liver abscess caused by non-Klebsiella pneumoniae and Klebsiella pneumoniae. J Microbiol Immunol Infect 2004;37:176-84.

[21] Singer ME, Applegate KE. Cost-effectiveness analysis in radiology. Radiology 2001;219:611-20.

[22] McDonough CM, Tosteson AN. Measuring preferences for cost-utility analysis: how choice of method may influence decision-making. Pharmacoeconomics 2007;25:93-106.

[23] Gazelle GS, McMahon PM, Beinfeld MT, Halpern EF, Weinstein MC. Metastatic colorectal carcinoma: cost-effectiveness of percutaneous radiofrequency ablation versus that of hepatic resection. Radiology 2004:233:729-39. 